the last follow-up visit was unrelated to age or the duration of SLE, but it correlated with the initial SLEDAI score (Spearman $p$ $<0.05$ ); the higher the initial SLEDAI score, the higher the SLICC score at the end of follow-up. The SLICC score was significantly lower (Mann Whitney $\mathrm{p}<0.05$ ) in patients with antiENA antibodies, particularly those who had anti-Ro/SS-A (mean 1.11 vs 1.79 ) or anti-La/SS-B antibodies (mean 0.52 vs 1.7 ). SLICC score showed no relation with anticardiolipin antibodies. The 8 patients who died in the course of the study had significantly higher SLICC scores (mean 2.87 vs 1.44).

Conclusion High SLEDAI score at the onset of SLE and the absence of anti-ENA antibodies, particularly anti-Ro/SS-A or anti-La/SS-B antibodies were associated with significantly higher SLICC scores, suggesting that these factors may indicate poor prognosis in SLE.

\section{FRI0123 CUMULATIVE TISSUE DAMAGE IN PATIENTS WITH JUVENILE-ONSET SYSTEMIC LUPUS ERYTHEMATOSUS}

I Monteagudo, FJ López-Longo, CM González, Al Turrión, CA Montilla, M Moreno-Zazo, N Caro, R Del Castillo, C González-Montagut, L Cebrián, E Carmona, L Carreño. Rheumatology, Hospital General Universitario Gregorio Marañón, Madrid, Spain

\subsection{6/annrheumdis-2001.158}

Background In earlier studies, we found that the juvenile onset of systemic lupus erythematosus (SLE) did not significantly modify the clinical expression of the disease. ${ }^{1}$ Although juvenileonset SLE may have a more sombre prognosis than adult-onset SLE, there have been few comparative studies of these types of SLE.

Objectives To compare the Systemic Lupus International Collaborating Clinics/American College of Rheumatology Damage Index (SLICC/ACR) of a cohort of patients with juvenile-onset SLE and patients with adult-onset SLE.

Methods We calculated the SLICC/ACR scores of 236 patients diagnosed as SLE between 1988 and 2000. The SLE disease activity index (SLEDAI) of the first hospital study was calculated retrospectively.

Results In 61 patients (25.8\%), SLE had begun before the age of 19 years. This group included 52 women (85.2\%). The mean age at onset of SLE was 13.4 years (SD 3.2) and mean duration of the disease was 9.9 years (SD 6.5). The comparison of patients with juvenile-onset SLE and a group of 175 patients with adult-onset SLE did not reveal significant differences in sex, duration of the disease, and SLEDAI score at the onset of the disease (mean 16.31; SD 8.26 vs 14.08; SD 8.08). There were no differences in the SLICC/ACR scores obtained at the last follow-up visit (mean 1.6; SD 1.7 vs 1.45 ; SD 1.94) (p > 0.05), although kidney disease (proteinuria over $3.5 \mathrm{~g} /$ day, treatment by dialysis or transplantation) (24 of $25 ; 39.3 \%$ vs 42 of 211 ; 24\%) (p: 0.03; OR: 2; 95\% CI: 1.1-3.8) and avascular necrosis (4 of 25 ; $6.6 \%$ vs 2 of 211 ; 1.1\%) (p: 0.04; OR: 6; 95\% CI: 1.08-3.4) were more frequent in patients with juvenile-onset disease. Both groups had a similar mortality ( 1 of $61 ; 1.6 \%$ vs 7 of $175 ; 4 \%)(\mathrm{p}>0.05)$.

Conclusion Although kidney disease and avascular necrosis were more frequent in patients with juvenile-onset SLE, the SLICC tissue damage score was similar in patients with juvenile-onset SLE and adult-onset SLE.

\section{REFERENCE}

1 Lupus 1999;8:287-92
FRI0124 DOES MIXED CONNECTIVE TISSUE DISEASE HAVE A LESS FAVOURABLE PROGNOSIS THAN SYSTEMIC LUPUS ERYTHEMATOSUS?

FJ López-Longo, CM González, Al Turrión, M Moreno-Zazo, R Del Castillo, N Caro, L Cebrián, C González-Montagut, E Carmona, S Gómez, I Monteagudo, L Carreño. Rheumatology, Hospital General Universitario Gregorio Marañón, Madrid, Spain

\subsection{6/annrheumdis-2001.159}

Background Mixed connective tissue disease (MCTD) has been considered to have a good prognosis, although some patients present severe clinical manifestations like pulmonary hypertension.

Objectives To compare the cumulative tissue damage occurring in patients with MCTD and systemic lupus erythematosus (SLE). Methods We compared the cumulative tissue damage index scores of patients with MCTD and SLE studied between 1988 and 2000. The consequences of MCTD and SLE and their treatment were evaluated using the Systemic Lupus International Collaborating Clinics/American College of Rheumatology Damage Index (SLICC/ACR) obtained at the last follow-up visit; scleroderma lesions were not taken into account in patients with MCTD.

Results We studied 37 patients with MCTD and 236 patients with SLE. There was no difference in the relative proportion of women in with MCTD and SLE (32 of 37; 86.5\% vs 211 of 236; 89.4\%). Mean age (40 years; SD 17.6 vs 39; SD 15.8), mean age at onset of disease (29 years; SD 17.6 vs 29; SD 14.7), and mean duration of disease (11 years; SD 7.9 vs 9.7; SD 6.9) were similar in both groups. There were no differences in mortality ( 1 of $37 ; 2.9 \%$ vs 8 of $236 ; 3.4 \%$ ) or SLICC score (1.43; SD 1.11 vs 1.49 ; SD 1.88). Patients with MCTD had more musculoskeletal sequelae (20 of $37 ; 54.1 \%$ vs 36 of 236 ; 15.3\%) (OR: 6.5) ( $\mathrm{p}<0.005)$ and pulmonary fibrosis (5 of 37; $13.5 \%$ vs 9 of $236 ; 3.8 \%$ ) (OR: 3.9$)$ (p < $<.05$ ) than patients with SLE. Patients with SLE had more kidney disease as defined by the SLICC (66 of $236 ; 28 \%$ vs 3 of $37 ; 8.1 \%$ ) ( $p<0.01$ ).

Conclusion In spite of the differences observed, the cumulative tissue damage and mortality were similar in MCTD and SLE, after excluding from consideration scleroderma lesions in patients with MCTD.

\section{FRI0125 CEREBRAL SINGLE-PHOTON EMISSION COMPUTED TOMOGRAPHY (SPECT) IN PATIENTS WITH SYSTEMIC LUPUS ERYTHEMATOSUS}

${ }^{1} \mathrm{CM}$ González, ${ }^{1} \mathrm{FJ}$ López-Longo, ${ }^{2} \mathrm{~A}$ Ortega, ${ }^{2} \mathrm{M}$ Suárez, ${ }^{1} \mathrm{~N}$ Caro, ${ }^{1} \mathrm{R}$ Del Castillo, ${ }^{1} \mathrm{C}$ González-Montagut, ${ }^{1} \mathrm{~L}$ Cebrián, ${ }^{2} \mathrm{Ml}$ Almoguera, ${ }^{1}$ I Monteagudo, ${ }^{1} \mathrm{~L}$ Carreño. ${ }^{1}$ Rheumatology; ${ }^{2}$ Nuclear Medicine, Hospital General Universitario Gregorio Marañón, Madrid, Spain

\subsection{6/annrheumdis-2001.160}

Background Hypoperfusion observed in cerebral SPECT studies of patients with SLE has been found to be related with clinical activity in 10 patients with SLE and no neuropsychiatric manifestations. ${ }^{1}$

Objectives To study the clinical significance of cerebral SPECT in unselected patients with SLE.

Methods We performed cerebral SPECT in 57 women with SLE seen consecutively in our clinic. In the same week that SPECT was performed, SLEDAI, ESR, the concentrations of anti-DNA antibody and the $\mathrm{C} 3$ and $\mathrm{C} 4$ complement fractions, and the SLICC/ACR tissue damage index were determined. 